\title{
Biomarkers for Diagnosis of Kawasaki Disease
}

\author{
Amit Rawat And SurJit Singh \\ From the Pediatric Allergy Immunology Unit, Advanced Pediatrics Center, PGIMER, Chandigarh, India. \\ surjitsinghpgi@rediffmail.com
}

$\mathrm{K}$ awasaki disease is an enigmatic condition characterized by vasculitis of medium-sized arteries, especially the coronaries. Majority age. The etiology of Kawasaki disease is still an enigma even though it was recognized almost 50 years ago [1]. If not diagnosed in time and not treated appropriately, approximately $15-25 \%$ of children afflicted with Kawasaki disease can develop coronary artery abnormalities. The diagnosis of Kawasaki disease is based on a set of clinical criteria, and there is no pathognomonic laboratory test for this condition. The clinical presentation of children with Kawasaki disease can be very variable and can test the clinical acumen of even the most astute pediatrician. Several children can present with incomplete and atypical forms of Kawasaki disease thereby further confounding the clinical differentiation between this disease and other febrile illnesses of childhood.

In recent years, considerable progress has been made in identifying disease susceptibility genes for Kawasaki disease, based on genome wide linkage and association studies [2,3]. Another set of studies have focused on the dysregulated cytokine networks in Kawasaki disease. Levels of several inflammatory cytokines and chemokines are found to be elevated during the acute phase of the disease. Wang, et al. [4] studied Th1 and Th2 cytokines in patients with Kawasaki disease. Levels of interleukin 6 , interleukin 20 , tumor necrosis factor- $\alpha$ (TNF- $\alpha$ ) and interferon- $\gamma$ were found to be elevated during the acute phase and showed a prompt decline following administration of intravenous immunoglobulin (IVIg). Further, the levels of TNF- $\alpha$ continued to remain elevated in patients who were resistant to IVIg or those with coronary artery abnormalities.

Interleukin 17 (IL-17) is a pro-inflammatory cytokine produced by a distinct subset of helper $\mathrm{T}$ cells known as $\mathrm{T}$ helper type 17 (Th17) cells. Th17 cells, and IL-17 produced by them, have a profound inflammatory action through upregulation of other cytokines and involvement of matrix metalloproteinases. Th17 cells have been incriminated in a host of autoimmune and allergic disorders. A derangement in relative proportions of Th17 and regulatory $\mathrm{T}$ cells (Treg) in patients with Kawasaki disease has been reported during the acute phase [5]. The proportion of Th17 cells was elevated whereas that of Treg was decreased during the acute phase. Th17 cell numbers were significantly higher in patients with Kawasaki disease resistant to IVIg compared to those who responded to immunoglobulin therapy [5].

Natriuretic peptides such as brain natriuretic peptides are synthesized by the ventricular myocytes in response to myocardial stress and injury in a wide variety of disorders such as congestive heart failure, acute coronary syndromes, and following major surgeries. Brain natriuretic peptide (BNP) is synthesized in a precursor form, PreproBNP, from the cardiac myocytes. It is first cleaved in the liver to form ProBNP which is further cleaved by an endopeptidase in the blood to form BNP and N-terminal pro-BNP (NTpro-BNP). NTpro-BNP is an inactive cleavage product which has a longer half-life in circulation than pro-BNP. Some previous studies have evaluated the diagnostic utility and the predictive value of NT-pro-BNP in Kawasaki disease [6,7]. The results from these studies have also been analyzed further in a recent systematic review and meta-analysis [8].

In this issue of Indian Pediatrics, Wu, et al. [9] have evaluated the diagnostic utility of NT-proBNP and IL-17 to differentiate incomplete Kawasaki disease from other febrile infectious illnesses of childhood. IL-17 and NTproBNP levels were estimated in 291 patients with complete Kawasaki disease, 74 patients with incomplete Kawasaki disease and 401 febrile infectious illnesses of unknown etiology. Combined with clinical criteria, when the cut-offs for IL-17 and NT-proBNP were set at 11.55 $\mathrm{pg} / \mathrm{mL}$ and $225.5 \mathrm{pg} / \mathrm{dL}$ respectively, the sensitivity and specificity of differentiating incomplete Kawasaki disease from infectious illnesses reached as high as $86.5 \%$ and $94.8 \%$, respectively.

Elevated levels of NT-proBNP are by no means specific for Kawasaki disease. The levels of NT-proBNP rise significantly in several heart ailments like congestive 
heart failure, acute coronary syndromes and congenital heart diseases. NT-proBNP levels are also dependent upon age. Infants less than 1 month of age tend to have higher levels than older children. Overall, it seems that the concurrent estimation of the biomarker NT-proBNP and IL-17 may serve as a useful adjunct for differentiation of Kawasaki disease, especially in its incomplete form, from other confounding infectious diseases of children.

Funding: None; Competing interests: None stated.

\section{REFERENCES}

1. Kawasaki T, Kosaki F, Okawa S, Shigematsu I, Yanagawa H. A new infantile acute febrile mucocutaneous lymphnode syndrome (MLNS) prevailing in Japan. Pediatrics. 1974;54:271-6.

2. Onouchi Y, Ozaki K, Burns JC, Shimizu C, Terai M, Hamada $\mathrm{H}$, et al. A genome-wide association study identifies three new risk loci for Kawasaki disease. Nat Genet. 2012;44:517-21.

3. Lee YC, Kuo HC, Chang JS, Chang LY, Huang LM, Chen MR. Two new susceptibility loci for Kawasaki disease identified through genome-wide association analysis. Nat
Genet. 2012;44:522-5.

4. Wang Y, Wang W, Gong F, Fu S, Zhang Q, Hu J, et al. Evaluation of intravenous immunoglobulin resistance and coronary artery lesions in relation to Th1/Th2 cytokine profiles in patients with Kawasaki disease. Arthr Rheum. 2013;65:805-14.

5. Jia S, Li C, Wang G, Yang J, Zu Y. The T helper type 17/ regulatory $\mathrm{T}$ cell imbalance in patients with acute Kawasaki disease. Clin Exp Immunol. 2010;162:131-7.

6. Dahdah N, Siles A, Fournier A, Cousineau J, Delvin E, Saint-Cyr C. Natriuretic peptide as an adjunctive diagnostic test in the acute phase of Kawasaki disease. Pediatr Cardiol. 2009;30:810-7.

7. Kawamura T, Wago M, Kawaguchi H, Tahara M, Yuge M. Plasma brain natriuretic peptide concentrations in patients with Kawasaki disease. Pediatr Int. 2000;42: 241-8.

8. Lin KH, Chang SS, Yu CW, Lin SC, Liu SC, Chao HY, et al. Usefulness of natriuretic peptide for the diagnosis of Kawasaki disease: A systematic review and meta-analysis. BMJ Open. 2015;5:e006703.

9. Wu L, Chen Y, Zhong S, Li Y, Dai X, Di Y. Blood Nterminal pro-brain natriuretic peptide and interleukin-17 for distinguishing incomplete Kawasaki disease from infectious diseases. Indian Pediatr. 2015;52:477-80. 


\title{
Duchenne Muscular Dystrophy: Advances in Molecular Genetics and Changing Strategies in Diagnosis, Prevention and Therapeutics
}

\author{
RekHa MitTal \\ From the Pediatric Neurology Unit, Max Superspeciality Hospital, Patparganj, New Delhi, India. \\ drrekhamittal@rediffmail.com
}

$\mathrm{D}$ uchenne Muscular dystrophy (DMD) is caused by a mutation of the dystrophin gene - the largest human gene, with 79 exons located at p21 on the $\mathrm{X}$ chromosome. Mutations of the dystrophin gene include deletions in $60 \%$ of the cases, duplications in $5-10 \%$ and point mutations in the rest [1]. A variation in the mutation can result in a milder form of the disease - Becker muscle dystrophy (BMD) - which has a later onset and much slower progression. Some patients with this mutation may have isolated cardiomyopathy. The dystrophin gene codes for the protein dystrophin, which is required for stabilization of the dystrophin-associated protein complex at the sarcolemma. It is the first protein to be characterized by reverse genetics, which means that the gene was discovered first and the protein was characterized thereafter [2]. Absence of dystrophin leads to destruction of the muscle fiber and progressive muscular weakness.

With the availability of molecular genetics techniques, the diagnostic workup of suspected DMD cases has been totally transformed. In this issue of Indian Pediatrics, Dey, et al. [3] have reported the genetic and clinical profile of patients diagnosed with DMD at a center in Eastern India. One hundred patients with a clinical diagnosis of DMD, and high Creatine phosphokinase (CPK) and myopathic electromyography (EMG) were evaluated for the dystrophin gene deletion; 73 tested positive. Eight out of nine patients, subjected to muscle biopsy with dystrophin staining of the muscle tissue, were confirmed to be DMD. The clinical features in the confirmed cases were studied; however, unfortunately, this study did not evaluate all the parameters in all cases. As expected, they did not find any correlation between the type/site of deletion and the clinical profile.

The clinical description of DMD, described in great clinical detail by Duchenne and Gower in the nineteenth century, remained almost unchanged for more than 100 years, till the description of the 'Valley sign' or 'Pradhan sign' in 1992 [4]. This sign describes a linear or oval depression over the posterior axillary fold, due to atrophy of the parts of the deltoid and infraspinatus muscles forming the posterior axillary fold, and hypertrophy of the adjacent muscle parts. This sign was found to be positive in $90 \%$ of cases of DMD, even when calf muscles were not hypertrophied (either due to early stage of the disease or in advanced disease) [5]. This sign was positive in $90 \%$ of patients in this study as well. The importance of clinical evaluation cannot be underestimated, especially in our country.

In DMD, CPK is raised manifold and the levels usually are in thousands, and the EMG shows myopathic changes. In this study, EMG was done in all patients, though, it needs to be emphasized that EMG is not required for evaluation of suspected DMD anymore. If the phenotype is characteristic, and if the $\mathrm{CPK}$ is high, one can straight away proceed for genetic testing [6,7]. However if the CPK is normal or mildly elevated, one may be dealing with Spinal muscle atrophy (SMA) type III, and only then an EMG may be done to look for neurogenic changes.

A routine muscle biopsy with a Hematoxylin Eosin (H\&E) staining may only show degeneration and regeneration of muscle fibres, proliferation of connective tissue and fatty infiltration, which is a picture not specific to DMD. Thus, a routine muscle biopsy with just H\&E staining is no longer recommended in any part of the workup of suspected DMD. However, when immunohistochemical staining is done for dystrophin, a complete absence of this protein suggests DMD, and a partial presence may be seen in patients with BMD. Muscle biopsy with dystrophin is thus the gold standard for the diagnosis of DMD. However, a muscle biopsy with dystrophin stain should be done only when the dystrophin gene mutation study is negative by the available methods [1], as has been done in this study.

The most easily available and common method for genetic studies for DMD diagnosis is the Multiplex PCR (the method used in the study) of the exons most 
commonly known to carry the mutations. It detects $98 \%$ of deletions, but does not pick up other mutations, and cannot be used for carrier detection [8]. A quantitative analysis of all exons can be done by multiplex ligation-dependent probe amplification (MLPA), which will also detect duplications as well as carriers [9]. However, this method is more expensive and not easily available, but may be required if the multiplex PCR is negative. Wherever possible, it must be done before doing a muscle biopsy. Recently, oligonucleotide-based Array comparitive genome hybridization (Array CGH) has been used for higher resolution analysis of the dystrophin gene; it may become the method of choice for the diagnosis of DMD in the future [10].

In the study reported in this issue of Indian Pediatrics, only 9 of the 27 genetically undiagnosed cases underwent muscle biopsy; 18 patients refused the procedure. This is not uncommon, as it is often perceived as a painful invasive procedure. Moreover, it is easier to get molecular diagnostic tests done as compared to muscle biopsy with various immunochistochemical stains. These 18 undiagnosed patients could have been either DMD with mutations other than deletion, not picked up by multiplex PCR, or they could be limb girdle muscle dystrophy. If muscle biopsy shows normal dystrophin, staining for dystrophin-associated proteins like sarcoglycans, dysferlin, calpain and others should be done to identify the type of dystrophy. Alternatively, the molecular diagnostic studies for other muscle dystrophies may be done, before carrying out a muscle biopsy in those patients testing negative for DMD gene [1].

The study cites 22 cases with affected siblings, showing that a large number of cases are diagnosed late, or that genetic diagnosis and counseling were not available. Early diagnosis, maternal carrier detection, carrier screening of female siblings, prenatal diagnosis and suitable genetic counseling, would prevent recurrence of DMD in families. Even though the whole process may be time consuming and expensive, the benefits of preventing this progressive disease, which at present has no cure, cannot be understated. Though, there is no cure for DMD but its progression can be slowed by the use of steroids (prednisolone or deflazacort). physiotherapy and rehabilitation.
Newer therapies being tried for DMD include gene therapy using viral vectors, exon-skipping methods, stem cell therapy (myoblast transfer), and delivery of dystrophin or compensatory proteins to the muscles $[11,12]$. Most of these therapies are likely to be successful only if started early.

Funding: None; Competing interests: None stated.

\section{REFERENCES}

1. Darras BT, Miller DT, Urion DK. Dystrophinopathies. In: Pagon RA, Adam MP, Ardinger HH, editors. Gene Reviews [Internet]. Seattle (WA): University of Washington, Seattle; 1993-2015. Available from: http://www.ncbi.nlm.nih.gov/ books/NBK1119/. Accessed May 5, 2015.

2. Kunkel LM, Beggs AH, Hoffman EP. Molecular genetics of Duchenne and Becker muscular dystrophy: Emphasis on improved diagnosis. Clin Chem. 1989;35(7 Suppl):B21-4.

3. Dey S, Senapati AK, Pandit A, Biswas A, Guin DS, Joardar $\mathrm{A}$, et al. Genetic and clinical profile of patients of Duchenne muscular dystrophy: Experience from a tertiary care center in Eastern India. Indian Pediatr. 2015;52:481-4.

4. Pradhan S. New clinical sign in Duchenne muscular dystrophy. Pediatr Neurol. 1994;11: 298-300.

5. Pradhan S. Valley sign in duchenne muscular dystrophy: Importance in patients with inconspicuous calves. Neurol India. 2002;50:184-6.

6. Paganoni S, Amato A. Electrodiagnostic evaluation of myopathies. Phys Med Rehabil Clin N Am. 2013;24:193207.

7. Bushby K, Finkel R, Birnkrant DJ, Case LE, Clemens PR, Cripe L, et al. Diagnosis and management of duchenne muscular dystrophy, part 1: diagnosis, and pharmacological and psychosocial management. Lancet Neurol. 2010;9:77-93.

8. Beggs AH, Koenig M, Boyce FM, Kunkel LM. Detection of $98 \%$ of DMD/BMD gene deletions by polymerase chain reaction. Hum Genet. 1990;86:45-8.

9. Verma PK, Dalal A, Mittal B, Phadke SR. Utility of MLPA in mutation analysis and carrier detection for Duchenne muscular dystrophy. Indian J Hum Genet. 2012;18:91-4.

10. Hegde MR, Chin ELH, Mulle JG, Okou DT, Warren ST, Zwick ME. Microarray-based mutation detection in the dystrophin gene. Hum Mutat. 2008.29:1091-9.

11. Goyenvalle A, Seto JT, Davies KE, Chamberlain J. Therapeutic approaches to muscular dystrophy. Hum Mol Genet. 2011;20(R1):R69-78.

12. Muir LA, Chamberlain JS. Emerging strategies for cell and gene therapy of the muscular dystrophies. Expert Rev Mol Med. 2009;11:e18. 